\title{
MODELLISTICA NUMERICA: IL PARADIGMA E LE ESPERIENZE AL POLITECNICO DI MILANO
}

\author{
ALFIO QUARTERONI (*)
}

SuNTO. - Questa presentazione intende fornire una sintetica rassegna dell'attività svolta nel Dipartimento di Matematica del Politecnico e nell'Istituto Lombardo in un quarto di secolo in ricerca nell'ambito dei modelli matematici e nelle loro applicazioni in seguito alla fondazione del MOX, Laboratorio di modellistica e calcolo scientifico. Inoltre illustro la collaborazione realizzata con diversi colleghi di altri Dipartimenti del Politecnico, con riferimento ad applicazioni in differenti campi della matematica computazionale. Infine accenno ad alcuni progetti sviluppati dal MOX a livello nazionale ed internazionale in questi ultimi 10 anni.

$$
* * *
$$

ABSTRACT. - In this presentation I have reported on the starting of numerical modelling at the Dipartment of Mathematics of the Politecnico of Milano in the early ninety's, followed by the foundation of MOX, the Laboratory of Modelling and Scientific Computing, back in 2002. I have then illustrated the collaborations carried out with many colleagues from several Departments at Politecnico di Milano focused on applications on different fields of computational mathematics, and reviewed some flagship projects that MOX has developed with industrial partners at both national and international level.

Questa breve presentazione intende fornire una rapida rassegna di quanto l'Istituto Lombardo e il Politecnico abbiano caratterizzato la mia esperienza di circa un quarto di secolo passato a Milano e le mie ricerche nell'ambito dei modelli matematici e delle loro applicazioni.

Arrivato nel 1989 al Dipartimento di Matematica, vi ho trovato diverse figure scientifiche dominanti nell'ambito delle applicazioni

(*) Istituto Lombardo Accademia di Scienze e Lettere, Politecnico di Milano, Italia. E-mail: alfio.quarteroni@epfl.ch 
della matematica: Luigi Amerio, analista raffinato, interessato alle applicazioni nell'ambito dei problemi di propagazione e alla loro risoluzione numerica. Carlo Cercignani, pioniere nell'analisi dell'equazione di Boltzman, della teoria cinetica e della risoluzione numerica di problemi di gas rarefatti. Laura Gotusso, cultore della teoria dell'approssimazione funzionale e delle sue applicazioni.

E poi Giovanni Prouse, Anna Zaretti, Sandro Salsa, Carlo Pagani, Marco Biroli, Claudio Citrini, cultori dell'analisi delle equazioni alle derivate parziali e del calcolo delle variazioni, declinate alla risoluzione di problemi di notevole interesse reale, quali i problemi di frontiera libera, le equazioni della dinamica dei fluidi, delle vibrazioni di strutture elastiche, di problemi inversi, solo per citarne alcuni.

Tutti questi colleghi hanno avuto un ruolo importante, in alcuni casi rilevantissimo, come noto, anche nell'Istituto Lombardo.

Naturalmente, essendo questo ambito di studio per sua stessa natura multidisciplinare, numerosi altri Dipartimenti e svariati altri gruppi di ricerca hanno dato in quegli anni contributi fondamentali allo sviluppo del Calcolo Scientifico in diversi ambiti applicativi.

Mi piace ricordarne alcuni, quelli con i quali ho avuto il piacere di collaborare o, semplicemente, di discutere: i colleghi Maier, Sacchi Landriani, Corradi dell'Acqua in analisi delle strutture, Gatti e Lacaita nella simulazione di dispositivi microelettronici, Diana nell'analisi di elementi finiti in vari ambiti relativi ai sistemi meccanici e ai problemi di vibrazione e deformazione di grandi strutture, Grandori e Faccioli per l'analisi della propagazione di onde sismiche, Gioda e Cividini per le simulazioni in ambito geotecnico, Rinaldi e Gatto per i sistemi dinamici e le applicazioni agli ecosistemi, Bittanti per i problemi di controllo e identificazione parametrica, Serazzi per l'analisi delle prestazioni dei calcolatori, Fumero e Pietrabissa per la biomeccanica, Borri, Mantegazza e Baron per le applicazioni in ambito aeronautico, Muzio, Osnaghi, Inzoli e Ricotti nella simulazione di problemi termofluidodinamici con applicazioni in vari settori dell'energia.

Naturalmente questo elenco è lungi dall'essere esaustivo.

In questi anni, mi è stata data l'opportunità di presentare i risultati di alcune mie ricerche nell'ambito dei modelli numerici all'Istituto Lombardo, in occasione dei tanti Convegni e Incontri di Studio che l'Istituto ha dedicato alla Matematica e alle sue applicazioni.

In particolare, mi è gradito segnalare che nell'ottobre 1998 ho presentato all'Istituto Lombardo per la prima volta i miei studi sulla 
modellistica del sistema vascolare, un'attività che ho poi sviluppato in modo intensivo sia a Milano sia a Losanna e che oggi vede impegnati una cinquantina di giovani ricercatori. Mi è inoltre gradito ricordare come nel febbraio $2004 \mathrm{mi}$ sia stato concesso il grande onore di tenere la Lezione Inaugurale del $201^{\circ}$ Anno Accademico dell'Istituto Lombardo.

Nel 2002 presso il Dipartimento di Matematica del Politecnico è stato fondato il laboratorio MOX con lo scopo di sviluppare la ricerca nell'ambito della modellistica matematica e del calcolo scientifico (con tecniche numeriche, probabilistiche e statistiche), integrarla nel percorso formativo del corso di laurea in Ingegneria Matematica, favorire la collaborazione scientifica interdipartimentale nel settore delle applicazioni della matematica, attrarre l'interesse del mondo industriale verso tematiche concernenti questa area della matematica moderna.

La motivazione principale muove dalla considerazione che ai nostri giorni algoritmi efficienti e computer potenti e di facile accessibilità consentono di affrontare (e risolvere!) problemi delle scienze di straordinaria complessità. Gli ambiti applicativi sono virtualmente illimitati e spaziano dall'ingegneria, alle scienze della vita e della salute, a quelle economiche e finanziarie, alla comprensione e gestione delle reti sociali.

A dodici anni dalla sua fondazione, il MOX ospita numerose decine di ricercatori e ha realizzato con successo più di un centinaio di progetti nei settori più svariati (dalla geofisica all'energia, dall'architettura urbana alla progettazione industriale, dalla medicina all'ambiente, ecc.), integrando le competenze nell'area della modellistica fisico-matematica, dell'analisi matematica, dell'analisi numerica e della statistica, della programmazione per il calcolo scientifico ad alte prestazioni.

Una componente rilevante del successo del MOX è la capacità di affrontare problemi di notevole complessità computazionale, depotenziandola e governandola, grazie alla messa a punto di diverse strategie di riduzione dimensionale (operanti sul modello matematico e/o sulla sua dimensione geometrica o algebrica), ispirate ai paradigmi dell' High Performance Computing (HPC) e del Large Scale Computation (LSC).

A titolo di esempio ci limitiamo a citare il caso dello studio del sistema cardiocircolatorio umano. In tale ambito i modelli matematici consentono di analizzare grandezze fisiche come pressioni, velocità, sforzi (quali lo shear stress sulla membrana endoteliale), assai problema- 
tiche da misurarsi in vitro e ancora di più in vivo, su geometrie reali ottenute con algoritmi di ricostruzione tridimensionale grazie al supporto delle moderne e non invasive tecnologie di acquisizione dei dati (ad esempio, la risonanza magnetica nucleare, l'angiografia digitale, la tomografia computerizzata, l'anemometria doppler).

La simulazione matematica dell'interazione fra flusso di sangue e deformazione della parete arteriosa richiede algoritmi che descrivano sia il trasferimento di energia a livello macroscopico tra il fluido (modellato tipicamente dalle equazioni di Navier-Stokes) e la parete (modellata con le equazioni della meccanica dei solidi), sia l'influenza a livello microscopico dello shear stress sull'orientamento, la deformazione e il danneggiamento delle cellule endoteliali. L'interazione fluido-parete dà luogo ad un complesso sistema di equazioni alle derivate parziali non lineari la cui approssimazione numerica richiede lo sviluppo di tecniche di precondizionamento che siano scalabili in ambiente di calcolo parallelo.

Nel contempo, le equazioni del flusso sono talvolta abbinate a modelli atti a descrivere il trasporto, la diffusione e l'assorbimento delle componenti chimiche in gioco (ad esempio ossigeno, lipidi, farmaci) nei diversi strati che compongono la parete delle arterie (intima, media e avventizia). Simulazioni numeriche di questo tipo possono aiutare a chiarire modificazioni biochimiche prodotte da alterazioni nel campo di flusso, dovute ad esempio alla presenza di una stenosi. Per dominare la complessità indotta anche dalla presenza di svariati parametri che caratterizzano il modello, tecniche di riduzione di modello, quali ad esempio la POD (principal order decomposition) o la RB (reduced basis) sono state messe a punto negli ultimi anni per consentire una efficace separazione fra onerosi calcoli offline ed agili calcoli online. Tecniche di questo genere consentono di abbattere drasticamente il costo computazionale anche in quei casi in cui la soluzione bigh-fidelity del problema originario richiede l'uso di svariate decine (talvolta centinaia) di milioni di incognite.

La complessità del modello aumenta ulteriormente qualora si passi da una descrizione locale a quella globale, in cui si tengano in considerazione le grandi e medie arterie (modellate con sistemi di equazioni mono-dimensionali) e la circolazione periferica (con modelli a parametri concentrati). Infine, integrando la funzionalità cardiaca, nelle sue componenti elettrica, meccanica e fluida, caratterizzate da molteplicità di scale sia spaziali sia temporali. Modellare matematicamente la funzione cardiaca è, da un lato, essenziale per "chiudere" il sistema circo- 
latorio fornendo le condizioni di pressione e portata alla radice dell'arco aortico. Dall'altro, diventa di fondamentale importanza per analizzare dal punto di vista matematico possibili disfunzioni del campo elettrico che si propaga nel miocardio, oppure le diverse patologie (congenite o sopraggiunte) legate al malfunzionamento delle valvole cardiache. I modelli di interazione elettro-meccanica sono particolarmente onerosi dal punto di vista computazionale. La loro risoluzione spesso richiede l'uso di algoritmi di tipo monolitico (globalmente accoppiati) con griglie di calcolo molto fini che conducono facilmente a sistemi nonlineari di centinaia di milioni di incognite.

Vogliamo inoltre ricordare come nel sistema circolatorio, condizioni di flusso separato, generazione di moti circolatori secondari a valle di biforcazioni (per esempio quella carotidea), aree a shear stress basso o temporalmente oscillante, sono oggi riconosciute quali potenziali fattori nello sviluppo di patologie arteriose. Una comprensione dettagliata del cambiamento emodinamico locale, degli effetti della modificazione delle pareti vascolari sullo schema del flusso, del graduale adattamento nel medio-lungo periodo del sistema globale a seguito di interventi chirurgici, oggi è reso possibile dall'uso di accurate simulazioni numeriche e spesso si rileva estremamente utile nella fase che precede la realizzazione di un trattamento terapeutico e/o chirurgico.

In effetti i modelli matematici sono anche usati per la chirurgia vascolare. Simulare il flusso in un bypass coronarico, in particolare la ricircolazione che si determina a valle del re-innesto nella coronaria può contribuire alla comprensione degli effetti della morfologia delle arterie sul flusso e quindi all'evoluzione post-chirurgica. Ogni anno l'8\% dei pazienti che si sottopongono all'intervento per l'impianto di un bypass rischiano la ri-occlusione (dopo 10 anni una significativa percentuale dei bypass impiantati devono essere sostituiti). La ripetizione di procedure di intervento chirurgico comporta un alto rischio di complicazioni: per questo molti aspetti devono essere compresi e controllati per evitare complicazioni e fallimenti post-operatori causati da ricircolazioni, flussi anomali e perturbati, ristenosi, iperplasia, etc.

Attualmente sono disponibili varie procedure di innesto e varie tipologie di bypass; la simulazione numerica applicata a strumenti matematici di ottimizzazione (individuate opportune grandezze da osservare e ottimizzare) favorisce la comprensione di fenomeni molto complessi all'interno del bypass e quindi una potenziale prevenzione di complicazioni, suggerendo configurazioni ottimizzate a vari livelli: dalla 
geometria locale (soprattutto nelle zone di innesto, dove si possono studiare varie forme a cuffia (Taylor, Miller)) alle grandezze che concorrono a costituire tutta la struttura del bypass (angolo di innesto, rapporto tra il diametro del bypass e dell'arteria nella zona di innesto, distanza tra il nuovo innesto e la stenosi, etc.).

La teoria del controllo ottimale e dell'optimal design può aiutare a "progettare" un by-pass che minimizzi la vorticità prodotta a valle del re-innesto nella coronaria. Analogamente, lo studio degli effetti delle protesi vascolari e degli impianti di valvole artificiali sull'emodinamica locale e globale può avanzare grazie a simulazioni sufficientemente accurate del campo di flusso del sangue.

La seconda fonte di perturbazone introdotta dagli stent è dovuta alla loro interazione con le cellule della parete vascolare con cui sono a contatto. Metalli come ferro, nichel etc., di cui sono composti alcune famiglie di stent, possono interagire con le cellule dell'intima e della media (gli strati che costituiscono la parete vascolare) causando una reazione infiammatoria che può portare ad una proliferazione incontrollata delle cellule muscolari lisce contenute nella media, riducendo il lume vascolare. Per contrastare questo fenomeno la ricerca è molto attiva nello sviluppo degli stent a rilascio di farmaco, rivestiti da un microstrato di materiale capace di immagazzinare e rilasciare lentamente un farmaco. I principali elementi di interesse sono la scelta del farmaco ed il design di una opportuna matrice capace di immagazzinare e rilasciare il farmaco scelto per lo stent. Inoltre, lo sviluppo di nuove microtecnologie consente di forare opportunamente i filamenti dello stent e di riempire i fori con strati di materiali o sostanze diverse. Questo apre la strada a innumerevoli possibilità per progettare uno stent con un profilo di rilascio predeterminato nel tempo di uno o più farmaci che possono interagire con la parete vascolare o con la superficie di contatto tra lo stent ed il sangue. A tal fine, la modellistica matematica e la simulazione al calcolatore permettono di valutare il comportamento di diverse configurazioni, rapidamente e con costi estremamente ridotti rispetto all'indagine sperimentale, mettendo in evidenza quali siano le soluzioni tecnologiche piu' efficaci.

Un ulteriore sviluppo in questa direzione è dato dall'applicazione delle tecniche di controllo ottimale. In questa cornice, un obiettivo non ancora raggiunto ma alla portata delle tecnologie progettuali e produttive e` quello di concepire una famiglia di stent a partire da un comune design geometrico diversificati per tipo di farmaco e profilo 
di rilascio, al fine di adattarsi in modo ottimale alle diverse patologie delle coronarie.

Per concludere, vale la pena osservare che non solo nella medicina computazionale ma in molti altri ambiti applicativi, la complessità computazionale richiede sempre più spesso il ricorso a tecniche di riduzione dimensionale. Tale riduzione si rivela cruciale nella risoluzione di equazioni alle derivate parziali parametrizzate, nei casi di problemi inversi o di identificazione, in problemi di cui si cerchino soluzioni approssimate in tempo reale e, ultimo ma non da ultimo, quando si passi dal caso deterministico a quello stocastico, ad esempio nell'analisi di quantificazione dell'incertezza, un'area che riscuote oggi un crescente interesse nel calcolo scientifico. 